\title{
Sub-Cell Shock Capturing for Discontinuous Galerkin Methods
}

\author{
Per-Olof Persson* and Jaime Peraire ${ }^{\dagger}$ \\ Massachusetts Institute of Technology, Cambridge, MA 02139, U.S.A.
}

\begin{abstract}
A shock capturing strategy for higher order Discontinuous Galerkin approximations of scalar conservation laws is presented. We show how the original explicit artificial viscosity methods proposed over fifty years ago for finite volume methods, can be used very effectively in the context of high order approximations. Rather than relying on the dissipation inherent in Discontinuous Galerkin approximations, we add an artificial viscosity term which is aimed at eliminating the high frequencies in the solution, thus eliminating Gibbs-type oscillations. We note that the amount of viscosity required for stability is determined by the resolution of the approximating space and therefore decreases with the order of the approximating polynomial. Unlike classical finite volume artificial viscosity methods, where the shock is spread over several computational cells, we show that the proposed approach is capable of capturing the shock as a sharp, but smooth profile, which is typically contained within one element. The method is complemented with a shock detection algorithm which is based on the rate of decay of the expansion coefficients of the solution when this is expressed in a hierarchical orthonormal basis. For the Euler equations, we consider and discuss the performance of several forms of the artificial viscosity term.
\end{abstract}

\section{Introduction}

The increase in computational power is enabling the simulation of multiple research problems previously deemed intractable such as unsteady Large Eddy Simulation and Aeroacoustics. These simulations, in turn, require highly accurate numerical methods which are efficient and yet robust to be applied to the simulation of practical problems.

Discontinuous Galerkin (DG) methods have gained increased popularity over recent years for the solution of the Euler and Navier-Stokes equations of gas dynamics. In principle, DG methods appear to combine in an optimal manner the shock capturing strategies well developed in the context of finite volume methods with the ability to employ high order discretizations on arbitrary unstructured meshes. In practice however, the natural dissipative mechanisms introduced by the DG methods, through the jump terms, are only sufficient to stabilize the solution in the presence of shocks when piecewise constant approximations are used. This can be understood be noting that in DG approximations of smooth solutions, the magnitude of the inter-element jumps, and hence the added dissipation, is proportional to $\mathcal{O}\left(h^{p+1}\right)$, where $h$ is a characteristic element dimension and $p$ the order of the interpolating polynomial. For higher order approximations, typically $p \geq 1$, explicit dissipation must be added to obtain stable solutions.

In order to be able to capture shocks with high order approximations, several approaches inspired by the finite volume methodology have been proposed. The most straightforward approach ${ }^{1,2}$ consists of using some form of shock sensing to identify the elements lying in the shock region and then reduce the order of the interpolating polynomial in those elements flagged by the sensor. Reducing the order of the interpolating polynomial increases the inter-element jumps and hence the amount of dissipation naturally added by the DG algorithm. If this reduction is taken all the way to piecewise constant interpolations, the method should

\footnotetext{
*Instructor, Department of Mathematics, MIT, 77 Massachusetts Avenue 2-363A, Cambridge, MA 02139. E-mail: persson@mit.edu. AIAA Member.

${ }^{\dagger}$ Professor, Department of Aeronautics and Astronautics, MIT, 77 Massachusetts Avenue 37-451, Cambridge, MA 02139. E-mail: peraire@mit.edu. AIAA Associate Fellow.
} 
be capable of handling any shock strength provided an appropriate Riemann solver is employed to calculate the inter-element fluxes.

Despite its simplicity, this approach may yield satisfactory answers, specially when combined with adaptive mesh refinement procedures. The ability to detect, in a robust way, the troubled elements is potentially an issue but several recipes that give acceptable answers can be found in the literature. The main drawback of this approach however, lies in the fact that reducing the order of the interpolating polynomial is equivalent to adding dissipation proportional to $\mathcal{O}(h)$ and therefore, the accuracy of the scheme is seriously degraded. An obvious solution, almost universally accepted, is that near the shocks, the solution can only be first order accurate and therefore, if one adaptively refines that region by locally reducing $h$, one may be able to alleviate the problem. A more serious problem however, stems from the fact that shocks are lower dimensional features which are strongly anisotropic. As a consequence, mesh adaptive strategies must incorporate some degree of directionality if they are to lead to an effective approach, specially in three dimensions. ${ }^{3}$ More sophisticated approaches exist for selecting the interpolating polynomial such as those based on weighted essentially non-oscillatory (WENO) concepts. ${ }^{4,5}$ These methods allow for stable discretizations near discontinuities while still maintaining a high order approximation. Although these methods have several attractive features, they appear to have a very high cost when the degree of the approximating polynomial is increased and to date have not yet been demonstrated in the practical unstructured mesh context. Other interesting alternatives, more closely related to the approach to be proposed here, are based on applying filters to the solution, ${ }^{6,7}$ the selective application of viscosity to the different spectral scales. ${ }^{8}$ Finally, we mention those methods based on reconstructing the solution from unlimited oscillatory solutions computed using a high order method. ${ }^{9,10}$ These methods hold the promise of yielding uniformly accurate solutions near the discontinuity in a pointwise sense. However, a number of issues still remain unresolved. For problems involving strong discontinuities, the unlimited solution is not stable and therefore some form of limiting is still required. Also, the extension of these methods to multiple dimensions is still an open question.

In this paper, we use a simple strategy which is inspired by the early artificial viscosity methods. ${ }^{11}$ This strategy is proving to be surprisingly effective in the context of high order DG methods. The rationale behind explicit artificial viscosity methods is to add viscosity to the original equations to spread discontinuities over a length scale that can be adequately resolved within the space of approximating functions. The goal is not to introduce discontinuities in the approximating space, but to resolve the sharp gradients existing in a viscous shock with continuous approximations. For low order approximations, such as piecewise constant and/or linear, this approach produces discontinuities which are spread over several cells (e.g. 2-4 cells) and therefore, it is considered to be inferior to the more established finite volume shock capturing approaches. This is because several cells are required to resolve a viscous shock profile with piecewise linear approximations.

However, for higher order polynomial approximations, the situation is different. The resolution given by a piecewise polynomial of order $p$ scales like $\delta \sim h / p$. This means that the amount of artificial viscosity required to resolve a shock profile is only $\mathcal{O}(h / p)$. Keeping $h$ fixed, the amount of artificial viscosity required scales like $1 / p$ and the accuracy of the solution in the neighborhood of the shock becomes $\mathcal{O}(h / p)$. This compares favorably to the more standard approaches which are only $\mathcal{O}(h)$ accurate. In other words, for high order $p$, we can exploit sub-cell resolution and obtain shock profiles which are much thinner than the element size. Recall that in the standard approach, the order of the interpolating polynomial is reduced over the whole element and as a consequence, there is no hope for resolving the shock profile at a sub-cell level.

Introducing viscosity to the original equations requires discretizing second order derivatives which is not straightforward when the approximating space is discontinuous. A number of methods have been proposed to deal with this situation, ${ }^{12}$ each of them having some merits and drawbacks. Here, we use the Local Discontinuous Galerkin (LDG) approach, ${ }^{13}$ but we expect that this choice is not critical to the approach described.

We note that in the proposed approach, no attempt is made at exploiting the DG natural stabilization since the inter-element jumps are always kept small. In fact, the magnitude of the jumps could be effectively used to determine the cells that require additional dissipation.

We claim that spreading the discontinuities over a thin boundary layer, that can be resolved by the underlying approximating space, has several important advantages from a computational viewpoint. For instance, shocks profiles can be accurately propagated through the grid without generating noise when element boundaries are crossed. We suspect this is particularly important in aeroacoustics applications. On the other hand, in optimal control applications requiring solution sensitivities, the presence of discontinuities presents a number of theoretical issues, which essentially disappear when the solution is regularized. Finally, 
spreading the discontinuities over a finite width produces discrete systems which are much better conditioned and easier to solve. ${ }^{14}$

\section{Proposed Approach for Scalar Conservation laws}

\section{A. DG Discretization}

We consider a conservation law of the form,

$$
\frac{\partial u}{\partial t}+\nabla \cdot \boldsymbol{F}=0
$$

defined over a domain $\Omega$, with suitable boundary conditions. Here, $u(\boldsymbol{x})$ is the conserved quantity and $\boldsymbol{F}(u)$ is the vector of fluxes in the spatial directions. We are assuming without loss of generality that we are working in two dimensions $\boldsymbol{x}=\left(x_{1}, x_{2}\right)$. This equation is discretized on a triangulation of the computational domain using the DG method with an interface flux function of the Roe or Lax-Friedrichs type. ${ }^{15}$ In our implementation, we use nodal shape functions and equally spaced nodes. ${ }^{16}$ This seems to be adequate provided the order of the interpolating polynomials is kept below about 7 . The time integration is performed either explicitly using a Runge-Kutta time-stepping or implicitly using a backward difference discretization of the time derivative and an iterative Newton method to solve within each time step. ${ }^{14}$

For smooth solutions the DG algorithm is found to perform very well allowing for approximations of arbitrary accuracy, which is determined by the order of the approximating polynomial. It is well known that, in the general non-linear case, the solution may develop discontinuities even if the initial and boundary data are smooth. Higher order numerical approximations to these discontinuous solutions exhibit Gibbstype oscillations which frequently lead to instability. In order to remedy this situation a special treatment is required.

\section{B. Model Term and LDG Discretization}

When the equation (1) is not purely hyperbolic but contains a small amount of viscosity, the shocks or discontinuities become thin layers where the solution changes rapidly. The idea behind the artificial viscosity approach $^{11}$ is to add a dissipative model term to the original equations of the form,

$$
\frac{\partial u}{\partial t}+\nabla \cdot \boldsymbol{F}=\nabla \cdot(\varepsilon \nabla u)
$$

Here, the parameter $\varepsilon$ controls the amount of viscosity. The shocks that may appear in the solution to this modified equation will spread over layers of thickness $\mathcal{O}(\varepsilon)$. Therefore, when attempting to approximate this solutions numerically, $\varepsilon$ should be chosen as a function of the resolution available in the approximating space. Near the shocks, we take $\varepsilon=\mathcal{O}(h / p)$, where $h$ is the element size and $p$ is the order of the approximating polynomial. Away from discontinuities, where the unmodified solution is resolved, we want $\varepsilon=0$.

We note that the discretization of the model term in equation (2) can not be carried out with the standard DG method due to the presence of the higher derivatives. Here, we choose the Local Discontinuous Galerkin approach described in ${ }^{13}$ to carry out the discretization of the viscous term. We presume that other schemes proposed to handle second order derivatives in the DG context could also be used. ${ }^{12}$ In order to apply the LDG method, equation (2) is first written as a system of first order hyperbolic equations by introducing the the auxiliary flux variables $\boldsymbol{q}$,

$$
\begin{aligned}
\frac{\partial u}{\partial t}+\nabla \cdot \boldsymbol{F}-\nabla \cdot \boldsymbol{q} & =0 \\
\boldsymbol{q}-\varepsilon \nabla u & =0 .
\end{aligned}
$$

A standard DG discretization can now be applied to this system of first order hyperbolic equations. By appropriately choosing the interface fluxes,$^{13}$ it is possible to obtain stable discretizations with optimal apriori error estimates using equal order interpolations for both $u$ and $\boldsymbol{q}$. The name Local Discontinuous Galerkin stems from the fact that, for certain numerical fluxes choices, it is possible to locally eliminate from the discrete system the unknowns corresponding to the additional variables $\boldsymbol{q}$. The final result is an algebraic system of equations which only involves the unknowns associated with the primal variable $u$. 
We note that the use of the LDG method for the model term adds a substantial amount of complexity and cost to the original DG discretization. Clearly, there is a temptation to ignore the inter-element contributions and just consider the diffusion operator applied within each element as one may obtain when using continuous approximations e.g. ${ }^{17}$ While being very attractive from the computational point of view, this approach is not really effective at eliminating the higher frequencies and in fact, it does the opposite. It tends to flatten out the solution within each element thus increasing the inter-element jumps.

\section{Discontinuity Sensor}

In order to determine a suitable sensor for discontinuities, we write the solution within each element in terms of a hierarchical family of orthogonal polynomials. In 1D, the solution is represented by an expansion in terms of orthonormal Legendre polynomials, whereas in $2 \mathrm{D}$, an orthonormal Koornwinder basis ${ }^{18}$ is employed within each triangle. For smooth solutions, the coefficients in the expansion are expected to decay very quickly. On the other hand, when the solution is not smooth, the strength of the discontinuity will dictate the rate of decay of the expansion coefficients. We express the solution of order $p$ within each element in terms of an orthogonal basis as

$$
u=\sum_{i=1}^{N(p)} u_{i} \psi_{i},
$$

where $N(p)$ is the total number of terms in the expansion and $\psi_{i}$ are the basis functions. In addition, we also consider a truncated expansion of the same solution, only containing the terms up to order $p-1$, that is

$$
\hat{u}=\sum_{i=1}^{N(p-1)} u_{i} \psi_{i}
$$

Within each element $\Omega_{e}$, the following smoothness indicator is defined,

$$
S_{e}=\frac{(u-\hat{u}, u-\hat{u})_{e}}{(u, u)_{e}}
$$

where $(\cdot, \cdot)_{e}$ is the standard inner product in $L_{2}\left(\Omega_{e}\right)$. Roughly speaking we aim for our approximate solution to be at least continuous. Therefore, in $1 \mathrm{D}$, the Fourier coefficients would decay at least like $\sim 1 / k^{2}$. Given that the sensor used involves squared quantities and assuming that the polynomial expansion has a similar behavior to the Fourier expansion, we expect that the value of $S_{e}$ will scale like $\sim 1 / p^{4}$. This expectation is confirmed in actual computations. In practice, we have found $S_{e}$ to be a remarkably reliable indicator

Once the shock has been sensed, the amount of viscosity is taken to be constant over each element and determined by the following smooth function,

$$
\varepsilon_{e}=\left\{\begin{array}{lll}
0 & \text { if } & s_{e}<s_{0}-\kappa \\
\frac{\varepsilon_{0}}{2}\left(1+\sin \frac{\pi\left(s_{e}-s_{0}\right)}{2 \kappa}\right) & \text { if } \quad s_{0}-\kappa \leq s_{e} \leq s_{0}+\kappa \\
\varepsilon_{0} & \text { if } \quad s_{e}>s_{0}+\kappa .
\end{array}\right.
$$

Here, $s_{e}=\log _{10} S_{e}$ and the parameters $\varepsilon_{0} \sim h / p, s_{0} \sim 1 / p^{4}$, and $\kappa$ is chosen empirically sufficiently large so as to obtain a sharp but smooth shock profile.

In our experience, the sensor described above performs better than alternative indicators based on the residual of the solution, ${ }^{19}$ but this is clearly still an open question.

\section{Extension to the Euler Equations}

We now consider the Euler equations in 2D,

$$
\frac{\partial \boldsymbol{U}}{\partial t}+\nabla \cdot \boldsymbol{F}=\mathbf{0}
$$

where, $\boldsymbol{U}=\left(\rho, \rho u_{1}, \rho u_{2}, \rho E\right)^{T}$ is the vector of conserved quantities and $\boldsymbol{F}=\left(\boldsymbol{F}_{1}, \boldsymbol{F}_{2}\right)$ is the generalized flux vector whose components are the fluxes of $\boldsymbol{U}$ along the spatial coordinate directions. Thus, $\boldsymbol{F}_{i}=$ 
$\left(\rho u_{i}, \rho u_{1} u_{i}+p \delta_{1 i}, \rho u_{2} u_{i}+p \delta_{2 i}, \rho u H\right)^{T}$ for $i=1,2$. In these expressions, $\rho$ is the fluid density, $u_{i}$ are the velocity components, $E$ is the internal energy, $p$ is the pressure, $H$ is the total enthalpy and $\delta_{i j}$ is the Kronecker symbol.

The application of the DG method to the Euler equations is straightforward. The only choice to be made is the particular form of the numerical flux employed. We have experimented with Roe ${ }^{20}$ and Lax-Friedrichs ${ }^{15}$ numerical flux formulae, but have found no significant differences when the order of the approximation is higher than, say, 2 or 3 . As expected, the DG discretization performs very well for smooth flows.

The extension of the shock capturing scheme described above for the scalar case to the Euler equation requires a viscosity model to smear out the discontinuities and a suitable discontinuity sensor. We have experimented with two approaches which are described below.

\section{A. Laplacian Artificial Viscosity}

First, we consider a model term of the form,

$$
\frac{\partial \boldsymbol{U}}{\partial t}+\nabla \cdot \boldsymbol{F}=\nabla \cdot(\varepsilon \nabla \boldsymbol{U})
$$

and expect that discontinuities will spread over a layer of thickness $\mathcal{O}(\varepsilon)$. The application of the LDG approach requires that we rewrite the above equations as a system of first order equations by introducing additional variables for the viscous fluxes

$$
\begin{aligned}
\frac{\partial \boldsymbol{U}}{\partial t}+\nabla \cdot \boldsymbol{F}-\nabla \cdot \boldsymbol{q} & =\mathbf{0} \\
\boldsymbol{q}-\varepsilon \nabla \boldsymbol{U} & =\mathbf{0} .
\end{aligned}
$$

In order to eliminate the effect of the added viscosity away from discontinuities we use the discontinuity sensor described above for the scalar case and apply it to a characteristic quantity such as density or Mach number. In this case $\varepsilon$, in equation (12) is replaced by an element-wise viscosity coefficient $\varepsilon_{e}$ which vanishes away from the discontinuities.

This approach works remarkably well in practice as illustrated by some of the examples presented below. We have found out however that, for high Mach numbers, the value of the viscosity coefficient needs to be increased to maintain stability and this results in wider shocks. We also point out that this approach is not very effective at discriminating between shocks and contact discontinuities across which the density might be discontinuous but the pressure and normal velocities are continuous.

\section{B. Physical Artificial Viscosity}

An alternative viscosity model is based on the real physical dissipation of an ideal gas. In this case we write,

$$
\frac{\partial \boldsymbol{U}}{\partial t}+\nabla \cdot \boldsymbol{F}=\nabla \cdot \boldsymbol{F}^{v}
$$

where $\boldsymbol{F}^{v}=\left(\boldsymbol{F}_{1}^{v}, \boldsymbol{F}^{v}{ }_{2}\right)$ is the generalized viscous flux vector and the viscous fluxes along the spatial coordinate directions are given by,

$$
\boldsymbol{F}_{i}^{v}=\left(\begin{array}{c}
0 \\
\tau_{1 i} \\
\tau_{2 i} \\
u_{1} \tau_{1 i}+u_{2} \tau_{2 i}-Q_{i}
\end{array}\right), \text { for } i=1,2
$$

The stresses $\tau_{i j}$ are given by

$$
\tau_{i j}=\mu\left[\left(\frac{\partial u_{i}}{\partial u_{j}}+\frac{\partial u_{j}}{\partial u_{i}}\right)-\frac{2}{3} \delta_{i j} \nabla \cdot \boldsymbol{u}\right], \quad \text { for } \quad i=1,2,
$$

and the heat flux is given by

$$
Q_{i}=-\kappa \frac{\partial T}{\partial x_{i}}, \quad \text { for } \quad i=1,2
$$


Here, $\mu$ and $\kappa$ are the viscosity and thermal conductivity parameters, respectively, $T$ is the temperature and $\boldsymbol{u}=\left(u_{1}, u_{2}\right)$ is the velocity vector. The viscosity coefficient as assumed to be a function of temperature and here, we simply take $\mu=\mu^{*}\left(T / T^{*}\right)^{0.5}$, where $\mu^{*}$ is the viscosity at the sonic temperature $T^{*}=2 T_{0} /(\gamma+1)$. $T_{0}$ denotes the stagnation pressure temperature and $\gamma=C_{p} / C_{v}$ is the ratio of specific heats at constant pressure and constant volume, respectively.

Once again, the equation (13) is transformed into a system on first order non-linear equations by introducing additional unknowns now for the gradient of $\boldsymbol{U}$. Thus we have,

$$
\begin{aligned}
\frac{\partial \boldsymbol{U}}{\partial t}+\nabla \cdot \boldsymbol{F}-\nabla \cdot \boldsymbol{F}^{v}(\boldsymbol{U}, \boldsymbol{q} ; \mu, \kappa) & =\mathbf{0} \\
\boldsymbol{q}-\nabla \boldsymbol{U} & =\mathbf{0},
\end{aligned}
$$

where, we have explicitly indicated that the viscous fluxes are a function of viscosity and thermal conductivity coefficients as well as $\boldsymbol{U}$ and $\boldsymbol{q}$, but not of their gradients.

Here, we have chosen to define the auxiliary variables $\boldsymbol{q}$ as the gradient of the conservative variables $\boldsymbol{U}$. Note that this makes equation (18) not only linear but also decoupled from component to component. The option of defining the auxiliary variables $\boldsymbol{q}$ directly as the viscous stresses and heat fluxes was deemed to be computationally less efficient. We point out that while the viscous stresses are linear in the velocity, they are non-linear in the conservative variables. We also note that all the additional variables that are generated by the LDG approach are eliminated locally.

\section{Mach number Scaling}

When using physical viscosity, the shock structure is well understood. In particular, it can be shown ${ }^{21}$ that the shock thickness $\delta_{s}$ is proportional to $\mu$. Furthermore, we have that

$$
\frac{\delta_{s} \Delta u \rho^{*}}{\mu^{*}} \approx 1
$$

Here, $\Delta u$ is the change in normal velocity across the shock and $\rho^{*}$ and $\mu^{*}$ are the values of the density and viscosity evaluated at sonic conditions. That is at $M=1, T=T^{*}$. The value of $\Delta u$ for a normal shock can be easily determined as a function of the upstream Mach number, $M_{\infty}$, and the upstream velocity $u_{\infty}$, as $\Delta u=2 u_{\infty}\left(M_{\infty}^{2}-1\right) /\left[(\gamma+1) M_{\infty}^{2}\right]$. The value of $\rho^{*}$ depends on the shock structure but can also be reasonably approximated a-priori as a function of the upstream density $\rho_{\infty}$ and Mach number.

Thus, if we want to capture a shock which has a thickness $\mathcal{O}(h / p)$, as determined by the approximating space, expression (19) tells gives us the appropriate amount of $\mu^{*}$ that must be added, as a function of the Mach number, to obtain the desired shock thickness.

The relative value of the viscosity and thermal conductivity coefficients is given by the Prandtl number $\operatorname{Pr}=\mu C_{p} / \kappa$. Based on the value of $\operatorname{Pr}$, we consider two particular viscosity models.

\section{Physical model with $\operatorname{Pr}=3 / 4$}

The first model corresponds to $\operatorname{Pr}=3 / 4$. This is actually very close to the Prandtl number encountered in air. It can be shown that this choice gives a Prandtl number of unity with respect to the bulk viscosity of which in turn results in the enthalpy of the flow being constant across the shock. We recall that for any other value of the Prandtl number, the enthalpy before and after the shock will be the same but will be different across the shock where dissipation mechanisms are active. The entropy, in this case, can be shown to vary non-monotonically across the shock. This is illustrated with the $1 \mathrm{D}$ computations presented in the results section.

\section{Physical model with $\operatorname{Pr}=0$}

The second choice corresponds to ignoring heat transfer effects, that is $\kappa=0$, or $\operatorname{Pr}=\infty$. In this case, the enthalpy is not constant across the shock but but on the other hand the entropy behaves monotonically. 


\section{Shock Sensors}

Since away from the shocks we want the artificial viscosity to vanish we use a shock sensor to detect the presence of discontinuities. We have found that for the physical model with $\operatorname{Pr}=3 / 4$, the entropy layer tends to be wider than that of the other variables. Therefore, using entropy as the key variable in our shock sensing procedure turns out to result in a robust solution. For the model with $\operatorname{Pr}=0$, entropy layers are found to be very thin and in this case enthalpy, which is not constant across the shock, appears to provide a very good alternative. After an element-wise shock sensor has been determined, the actual viscosity used in the computation is determined according to an expression of the form (8) where the value of $\mu^{*}$ is used to specify $\varepsilon_{0}$.

\section{Results}

Our first example is the inviscid Burgers' equation with initial condition $u(x)=1 / 2+\sin 2 \pi x$ and periodic boundary conditions on the interval $[0,1]$. We discretize the PDE using the DG method with interpolation polynomials of degree $p=10$. The amount of artificial viscosity is determined by a Legendre decomposition of the solution within each element, and the viscous contribution to the PDE is discretized using the LDG method. Here, we integrate explicitly in time using a fourth-order Runge-Kutta solver.

The results at times $T=0.25$ and $T=0.50$ are shown in figure 1 . The shock is well approximated by the smooth high-order polynomials, and the entire shock is contained within about one element. The artificial viscosity does not introduce jumps between the elements, and the shock is accurately detected by the Legendre indicator as it propagates in time. Note that for a shock capturing scheme that produces jumps, the indicator will detect high frequencies even after the shock has propagated out of the element. Also, when the shock crosses between elements (as for $T=0.50$, right plots) the amount of required viscosity is typically small.
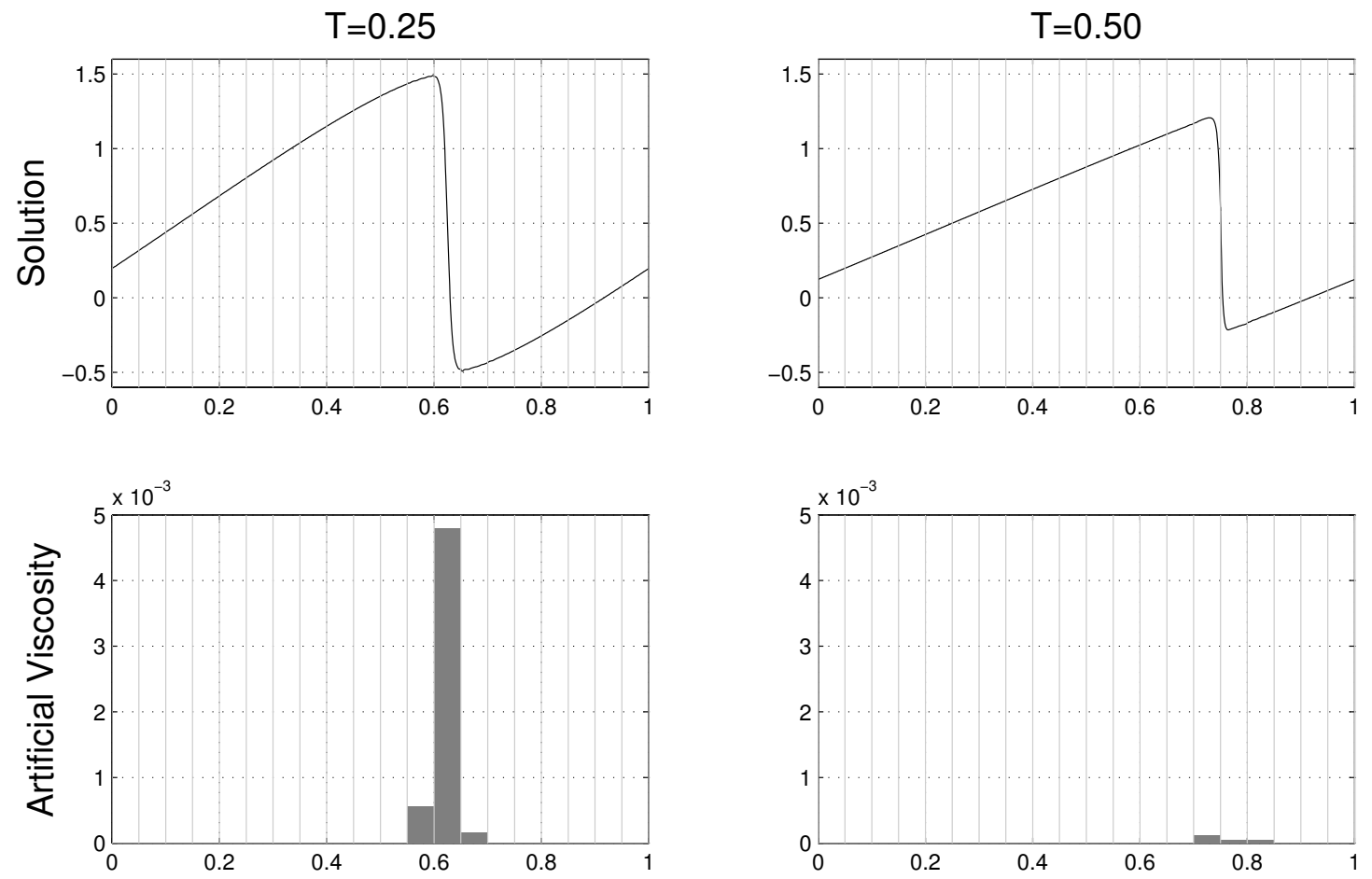

Figure 1. Solution of the inviscid Burgers' equation. The interpolation order is $p=10$, and the vertical lines indicate the element boundaries. Note how the shock is well-resolved within one element, and that the jumps between the elements are small.

In our second example we consider the 1D Euler equations. We solve the problem of a stationary shock in the unit domain using our LDG algorithm with 4 elements and $p=8$ polynomial approximations. Since 
the position of the shock is not uniquely determined, we arbitrarily require that the value of the density at the center of the domain be the average of the values before and after the shock. The equations are solved implicitly using a Newton method.

The purpose of this example is to compare the behavior of the different artificial viscosity models proposed. In particular, we consider the model described in section III.A based on a Laplacian form of the viscosity term as well as the two physical models described in section III.B for $\operatorname{Pr}=3 / 4$ and $\operatorname{Pr}=\infty$.

Figure 2 shows the computations using the different models and flow conditions. For the Laplacian viscosity model, the value of the viscosity coefficient has been tuned for each Mach number to obtain the sharpest possible solution while avoiding any significant overshoots. For the physical models a single constant has been determined and then, the Mach number scaling described above has been employed to determine the value of the viscosity coefficient for each flow condition.

The first observation is that the model based on Laplacian viscosity tends to give considerably wider shocks than the other two models. This is particularly true for the density variable. The Mach number profiles are rather similar in thickness for the three models although they are significantly shifted in space. We also note that the Laplacian model gives the sharper entropy profiles. This seems to indicate that entropy is probably not a robust key variable to detect shocks when using the Laplacian dissipation term.

Of the two physical models, the one corresponding to $\mathrm{Pr}=3 / 4$ seems to offer the best overall performance. Moreover, it has the added advantage that the enthalpy across the shock is constant. Its performance for high Mach number flows is clearly superior to the Laplacian model. Also, we note that because of the large overshoot in entropy, the entropy boundary layer is considerably thicker than with the other two models. This makes entropy a good candidate to be used as a shock detector indicator for this model.

In table 1, we give the numerical values of the shock thickness computed with the three models for each flow condition and variable. The thickness has been determined automatically according to the criterion that outside the layer the variations in the solution are less than $1 \%$.

We note that for this example, the element length is $h=0.25$ which illustrates the fact that all models are producing a shock which is thinner than the element length.

\begin{tabular}{|c|c|c|c|}
\hline \multicolumn{4}{|c|}{ Laplace } \\
\hline \hline$M_{\infty}$ & 2 & 5 & 10 \\
\hline$\rho$ & 0.31 & 0.31 & 0.34 \\
\hline$M$ & 0.28 & 0.25 & 0.25 \\
\hline$s$ & 0.16 & 0.16 & 0.19 \\
\hline$H$ & 0.39 & 0.37 & 0.43 \\
\hline
\end{tabular}

\begin{tabular}{|c|c|c|c|}
\hline \multicolumn{4}{|c|}{ Physical } \\
\hline \hline$M_{\infty}$ & 2 & 5 & 10 \\
\hline$\rho$ & 0.16 & 0.21 & 0.22 \\
\hline$M$ & 0.19 & 0.27 & 0.28 \\
\hline$s$ & 0.22 & 0.27 & 0.27 \\
\hline$H$ & N/A & N/A & N/A \\
\hline
\end{tabular}

\begin{tabular}{|c|c|c|c|}
\hline \multicolumn{4}{|c|}{ Physical $(\kappa=0)$} \\
\hline \hline$M_{\infty}$ & 2 & 5 & 10 \\
\hline$\rho$ & 0.22 & 0.24 & 0.22 \\
\hline$M$ & 0.24 & 0.25 & 0.27 \\
\hline$s$ & 0.15 & 0.16 & 0.15 \\
\hline$H$ & 0.34 & 0.36 & 0.28 \\
\hline
\end{tabular}

Table 1. Normalized shock thickness for density, Mach number, entropy and enthalpy using the three different viscosity models as a function of the upstream Mach number.

Next, we show our shock capturing scheme for two-dimensional Euler flows on a NACA0012 airfoil. In figure 3 we show the steady-state solution for a flow at Mach 0.8 with an attack angle of 1.5 degrees. We discretize the Euler equations using DG with fourth order polynomials, $p=4$, and apply the Laplacian artificial viscosity using the LDG method. The element-wise shock indicator is based on the components of the Koornwinder decomposition of the density $\rho$, and equal amounts of viscosity are added to all the equations.

In order to solve for a well-defined steady-state solution, the amount of viscosity we add is a smooth function of the solution. The entire viscous terms, as discretized by LDG, can then be linearized with respect to the solution, and we can use Newton's method to solve the equations. The terms that our shock capturing scheme introduces are highly non-linear, and to find the steady-state solution we integrate in time using the implicit backward Euler method with adaptive step size control. The full Jacobians are computed and stored as sparse matrices, and the linear systems are solved using a direct solver.

Again, we see how the shock is well-resolved within only one element. Note that we use a very coarse mesh (except at the tip of the airfoil), and we have chosen not to apply any $h$-adaptation around the shock to illustrate the sub-cell resolution. The entropy plot shows that the dissipation from the high-order scheme is very low. The Koornwinder indicator identifies the elements around the shock, and no viscosity is added to the elements further away. 


\section{Mach 2}

Density
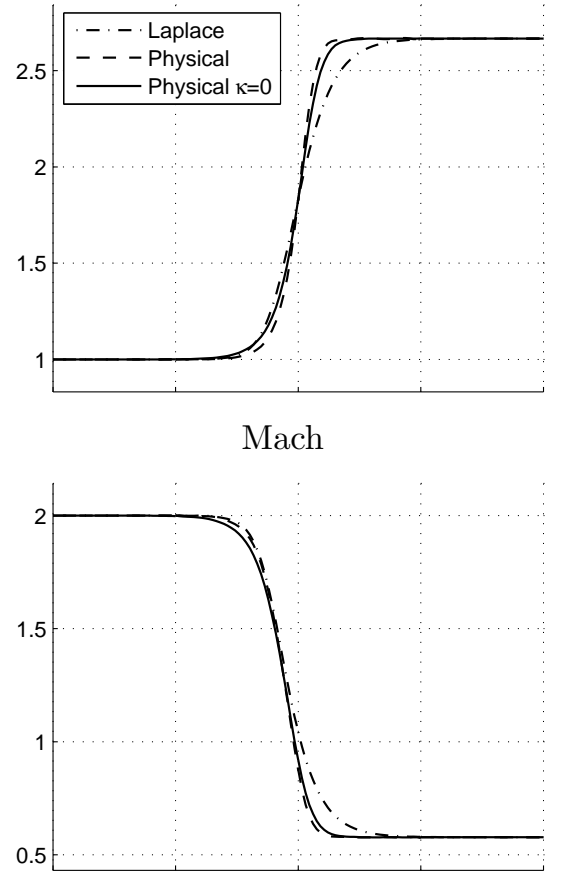

Entropy

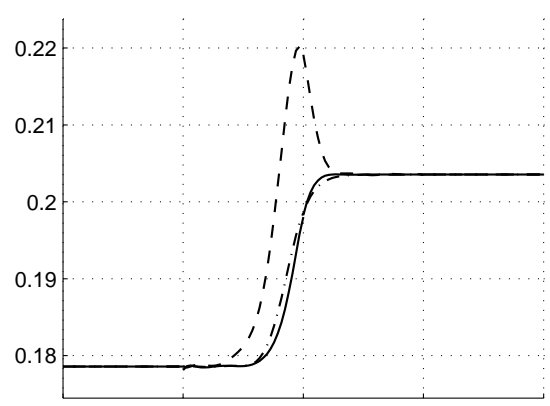

Enthalpy

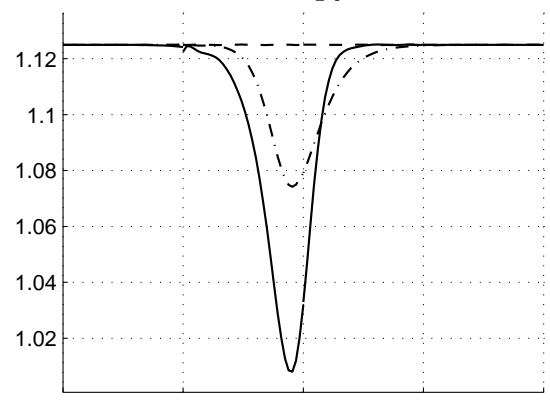

Mach 5

Density
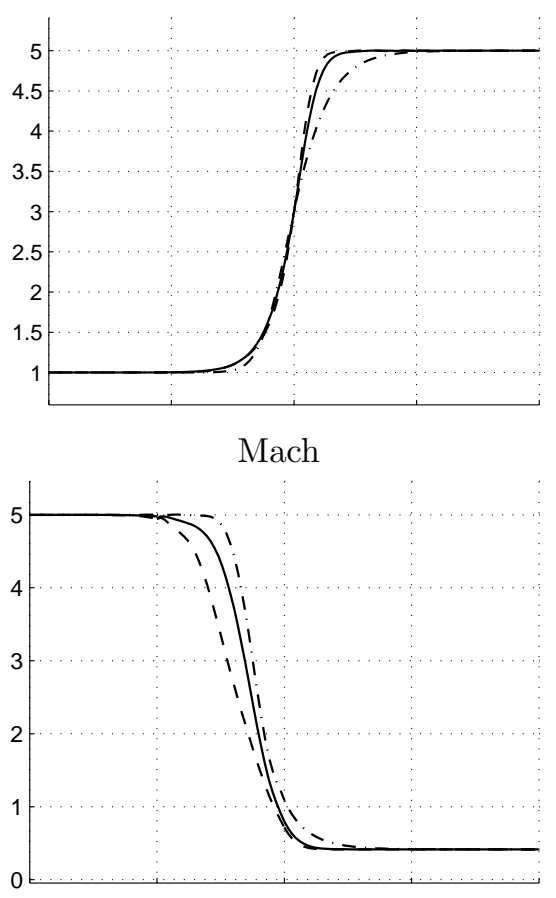

Entropy

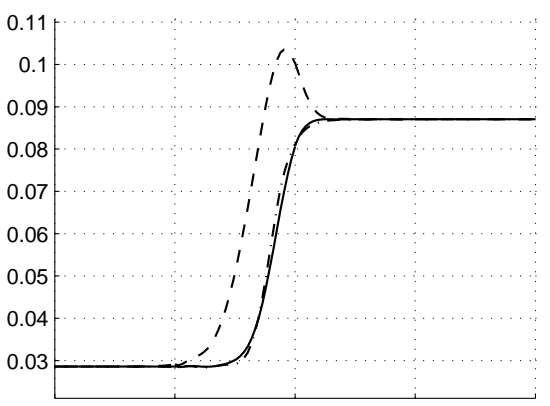

Enthalpy

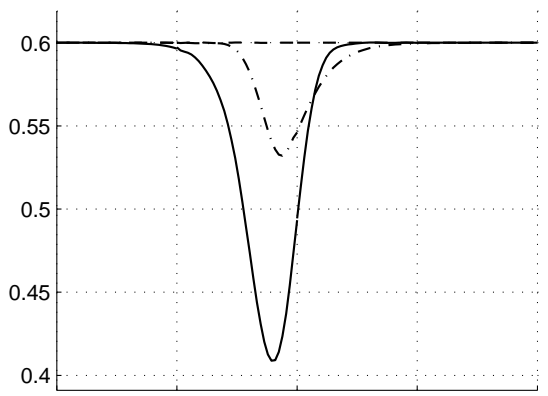

Mach 10

Density
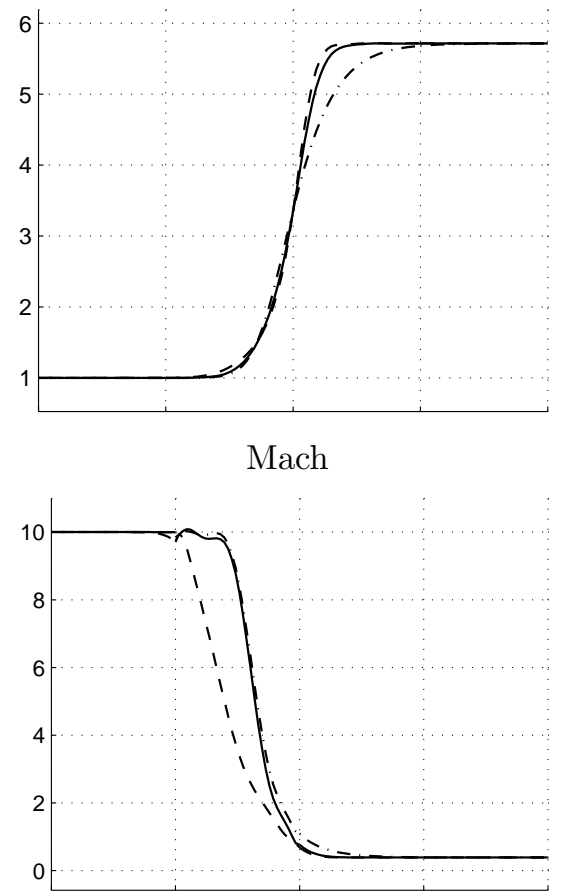

Entropy

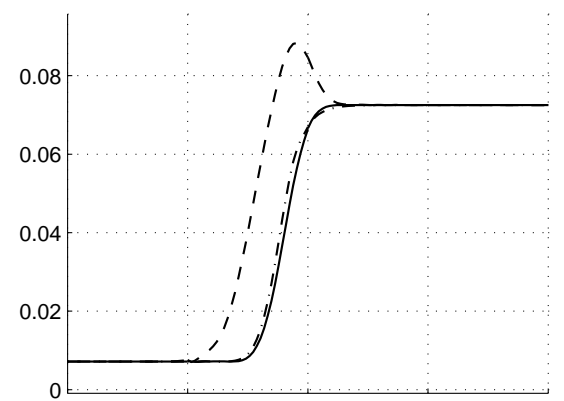

Enthalpy

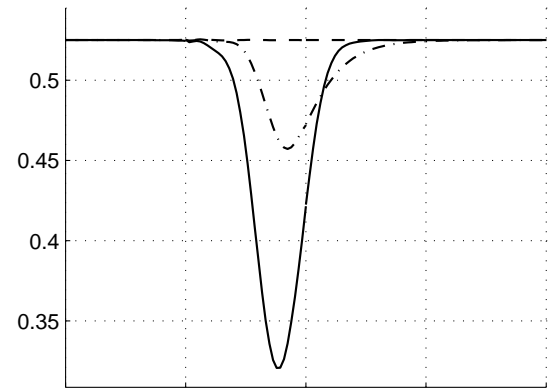

Figure 2. Computed shock profiles of density, Mach number, entropy and enthalpy using the three different viscosity models for three different upstream Mach numbers. 

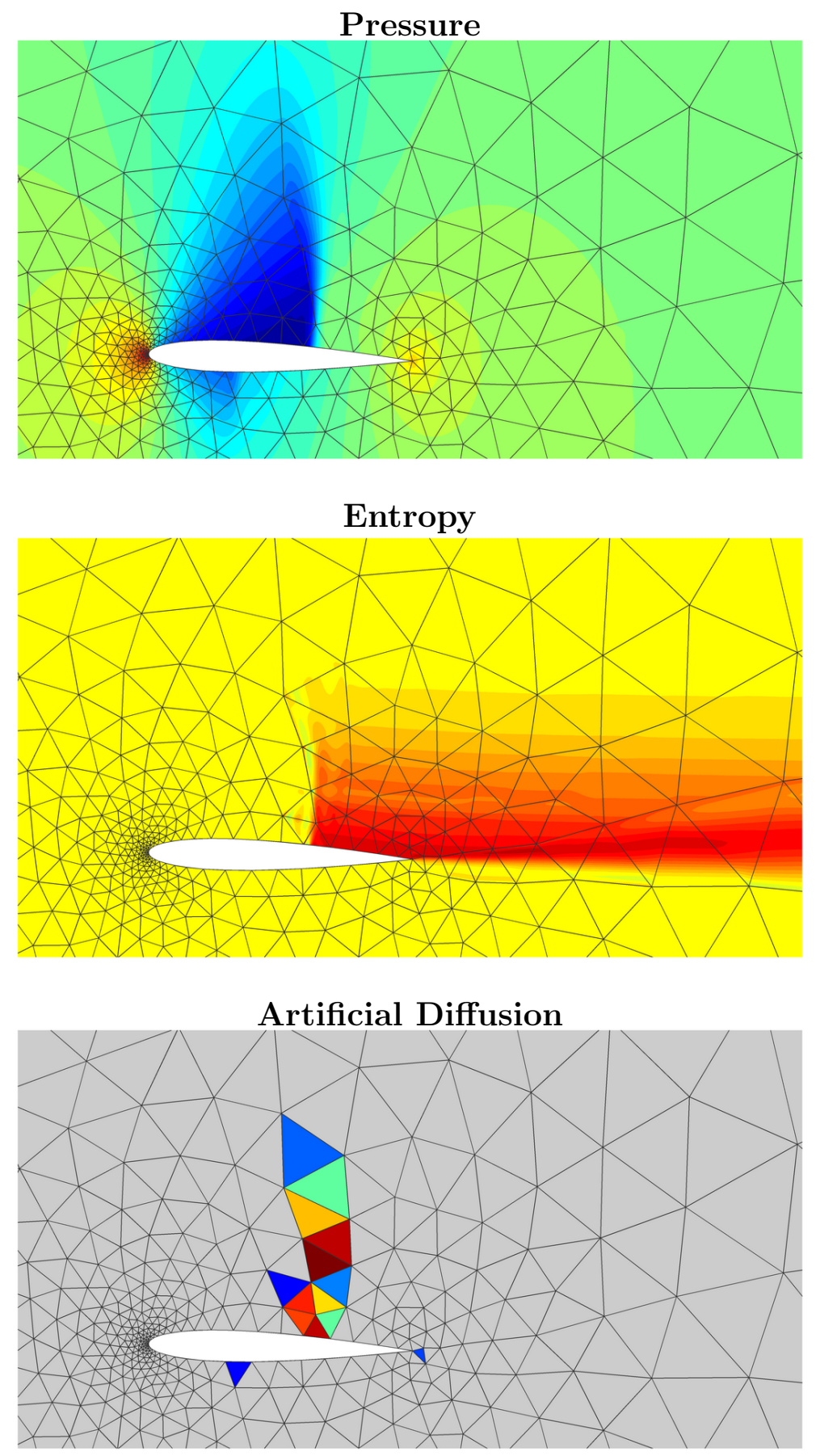

Figure 3. Transonic flow around a NACA0012 airfoil at Mach 0.8 , with interpolation of degree $p=4$. Note how the shock is accurately resolved within the elements. The high order scheme provides low dissipation away from the shock, as can be seen in the entropy plot (middle). The bottom plot shows the applied diffusion, with gray color representing no diffusion added. 
We also show an example of supersonic flow at $M=1.5$ around a NACA0012 in figure 4 computed with LDG and Laplacian viscosity. The non-linearities are now even stronger but the adaptive time stepping scheme finds the solution without any modifications. The advantage of having a smooth dependence on the solution is obvious in this problem. If, for example, the applied viscosity is a step function (or if the shock capturing scheme is discrete as with order reduction), the indicator changes between each Newton iteration making it hard to find a stationary solution.
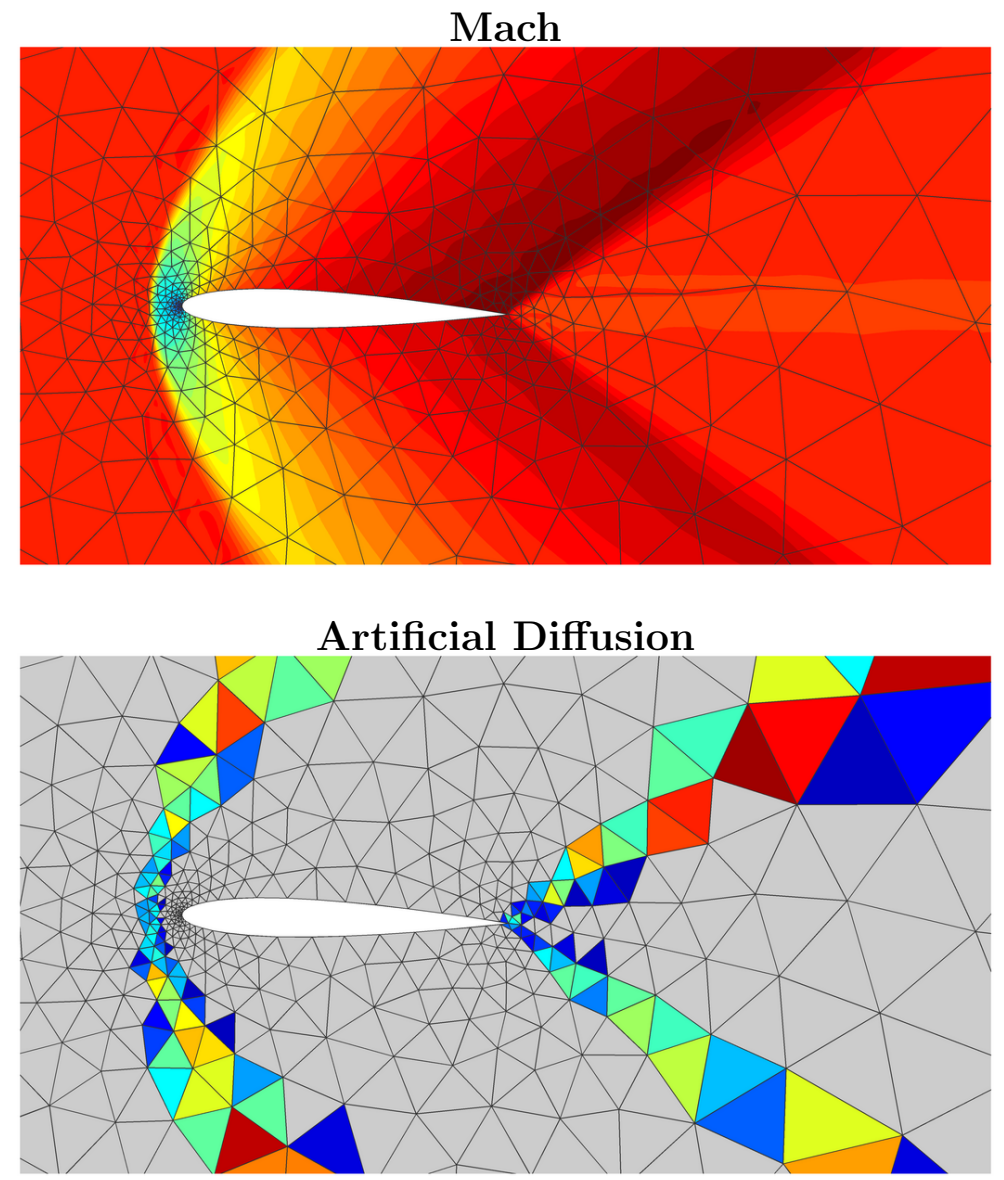

Figure 4. Supersonic flow around a NACA0012 airfoil at Mach 1.5, with interpolation of degree $p=4$. Again we see how the shocks are resolved within the elements, and the diffusion is only applied to elements around the shocks.

The final example consists of a flow about a cylinder at free stream Mach numbers of 2 and 5 . We purposely use a very coarse grid to illustrate the capabilities of the proposed approach. Here, we have used polynomial approximations of order $p=5$. The LDG method with a physical viscosity model with $\operatorname{Pr}=3 / 4$ has been used to capture the stronger shocks. The shock indicator in both cases has been determined based on the entropy. The system of equations has been solved implicitly using Newton's method. ${ }^{14}$ We note however that, specially for the Mach 5 case, the convergence of Newton's method is very much dependent on obtaining a good initial condition. We have used a continuation approaches in Mach number or determine a good initial guess for the iterative solver.

Even though the grids are very coarse, we note that the shock structure is well resolved and contained within an element. We also note that the shock thicknesses for both flow conditions are less than the element size, thus indicating that the method has a good behavior for high Mach numbers.

We point out that in practical situations, it is advantageous to integrate the equations implicitly. This is because the artificial viscosity added often places a severe restriction on the time step size, specially for high order approximations. 


\section{Conclusions}

We have presented a practical approach to shock capturing using DG approximations. We believe this is an important ingredient to making higher order methods viable in important research applications. In this paper, we have not demonstrated the use of $h$-adaptivity. It is clear that the use of $h$-adaptation will be beneficial in further narrowing the shock layers. How to optimally combine $p$ and $h$-adaption in the presence discontinuities, is still an open question. An optimal strategy in this case should not only consider the approximation aspects but also the algorithmic details of the implementation.

\section{Acknowledgements}

The authors would like to express their gratitude to the Singapore-MIT Alliance for the partial support of this work.

\section{References}

${ }^{1}$ Baumann, C. E. and Oden, J. T., "A discontinuous $h p$ finite element method for the Euler and Navier-Stokes equations," Int. J. Numer. Methods Fluids, Vol. 31, No. 1, 1999, pp. 79-95, Tenth International Conference on Finite Elements in Fluids (Tucson, AZ, 1998).

${ }^{2}$ Burbeau, A., Sagaut, P., and Bruneau, C.-H., "A problem-independent limiter for high-order Runge-Kutta discontinuous Galerkin methods," J. Comput. Phys., Vol. 169, No. 1, 2001, pp. 111-150.

${ }^{3}$ Dervieux, A., Leservoisier, D., George, P.-L., and Coudière, Y., "About theoretical and practical impact of mesh adaptation on approximation of functions and PDE solutions," Internat. J. Numer. Methods Fluids, Vol. 43, No. 5, 2003, pp. 507-516, ECCOMAS Computational Fluid Dynamics Conference, Part I (Swansea, 2001).

${ }^{4}$ Shu, C.-W. and Osher, S., "Efficient implementation of essentially nonoscillatory shock-capturing schemes," J. Comput. Phys., Vol. 77, No. 2, 1988, pp. 439-471.

${ }^{5} \mathrm{Shu}, \mathrm{C} . \mathrm{-W}$. and Osher, S., "Efficient implementation of essentially nonoscillatory shock-capturing schemes. II," J. Comput. Phys., Vol. 83, No. 1, 1989, pp. 32-78.

${ }^{6}$ Lomtev, I., Quillen, C. B., and Karniadakis, G. E., "Spectral/ $h p$ methods for viscous compressible flows on unstructured 2D meshes," J. Comput. Phys., Vol. 144, No. 2, 1998, pp. 325-357.

${ }^{7}$ Kanevsky, A., Carpenter, M., and Hesthaven, J. S., "Time Consistent Filtering in Spectral and Spectral Element Methods," J. Comput. Phys., 2005, (submitted).

${ }^{8}$ Tadmor, E., "Shock capturing by the spectral viscosity method," Comput. Methods Appl. Mech. Engrg., Vol. 80, No. 1-3, 1990, pp. 197-208, Spectral and high order methods for partial differential equations (Como, 1989).

${ }^{9}$ Hesthaven, J.S. Kaber, S. and Lurati, L., "Pade-Legendre Interpolants for Gibbs Reconstruction," J. Sci. Comput., 2005, (to appear).

${ }^{10}$ May, G. and Jameson, A., "High-Order Accurate Methods for High-Speed Flow," 17th AIAA Computational Fluid Dynamics Conference, Toronto, Ontario, June 2005, AIAA-2005-5252.

${ }^{11}$ Von Neumann, J. and Richtmyer, R. D., "A method for the numerical calculation of hydrodynamic shocks," J. Appl. Phys., Vol. 21, 1950, pp. 232-237.

${ }^{12}$ Arnold, D. N., Brezzi, F., Cockburn, B., and Marini, L. D., "Unified analysis of discontinuous Galerkin methods for elliptic problems," SIAM J. Numer. Anal., Vol. 39, No. 5, 2001/02, pp. 1749-1779 (electronic).

${ }^{13}$ Cockburn, B. and Shu, C.-W., "The local discontinuous Galerkin method for time-dependent convection-diffusion systems," SIAM J. Numer. Anal., Vol. 35, No. 6, 1998, pp. 2440-2463 (electronic).

${ }^{14}$ Persson, P.-P. and Peraire, J., "An Efficient Low Memory Implicit DG Algorithm for Time Dependent Problems," $44 t h$ AIAA Aerospace Sciences Meeting and Exhibit, Reno, Nevada, 2006, AIAA-2006-0113.

${ }^{15}$ Cockburn, B. and Shu, C.-W., "Runge-Kutta discontinuous Galerkin methods for convection-dominated problems," J. Sci. Comput., Vol. 16, No. 3, 2001, pp. 173-261.

${ }^{16}$ Hesthaven, J. S. and Warburton, T., "Nodal high-order methods on unstructured grids. I. Time-domain solution of Maxwell's equations," J. Comput. Phys., Vol. 181, No. 1, 2002, pp. 186-221.

${ }^{17}$ Brooks, A. and Hughes, T., "Streamline upwind/Petrov-Galerkin formulations for convection dominated flows with particular emphasis on the incompressible Navier-Stokes equations," Comput. Methods Appl. Mech. Engrg., Vol. 32, No. 1-3, 1982, pp. 199-259, FENOMECH '81, Part I (Stuttgart, 1981).

${ }^{18}$ Koornwinder, T. H., "Askey-Wilson polynomials for root systems of type BC," Hypergeometric functions on domains of positivity, Jack polynomials, and applications (Tampa, FL, 1991), Vol. 138 of Contemp. Math., Amer. Math. Soc., Providence, RI, 1992, pp. 189-204.

${ }^{19}$ Hughes, T. J. R. and Mallet, M., "A new finite element formulation for computational fluid dynamics. IV. A discontinuitycapturing operator for multidimensional advective-diffusive systems," Comput. Methods Appl. Mech. Engrg., Vol. 58, No. 3, 1986, pp. 329-336.

${ }^{20}$ Roe, P. L., "Approximate Riemann solvers, parameter vectors, and difference schemes," J. Comput. Phys., Vol. 43, No. 2, 1981, pp. 357-372.

${ }^{21}$ Liepmann, H. and Roshko, A., Elements of Gas Dynamics, John Wiley \& Sons, Inc., 1957. 

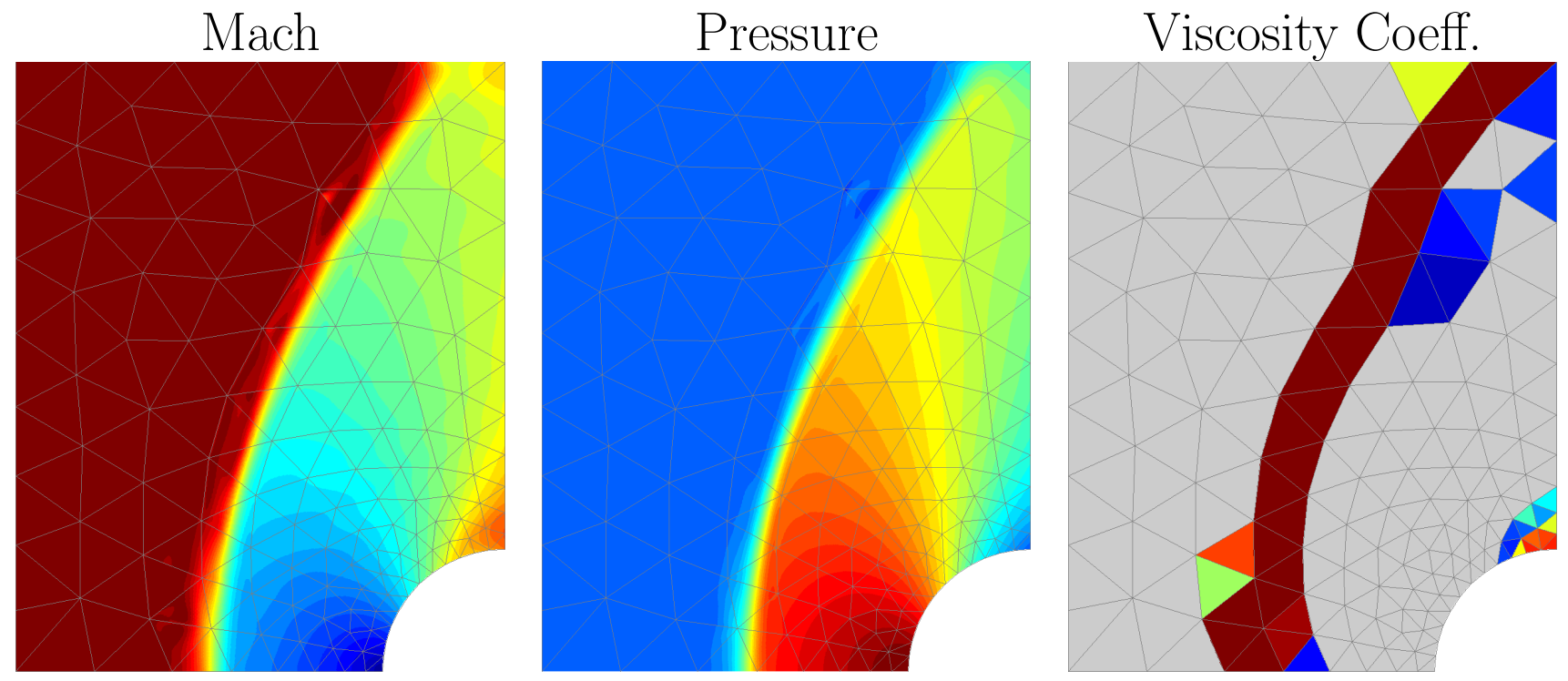

Figure 5. Mach 2 flow around a cylinder, with interpolation of degree $p=5$. Note that the coarse grid shown is the actual grid used in the computation. Some of the oscillation observed are due to the coarseness of the grid. We observe that the shocks are resolved within the elements.
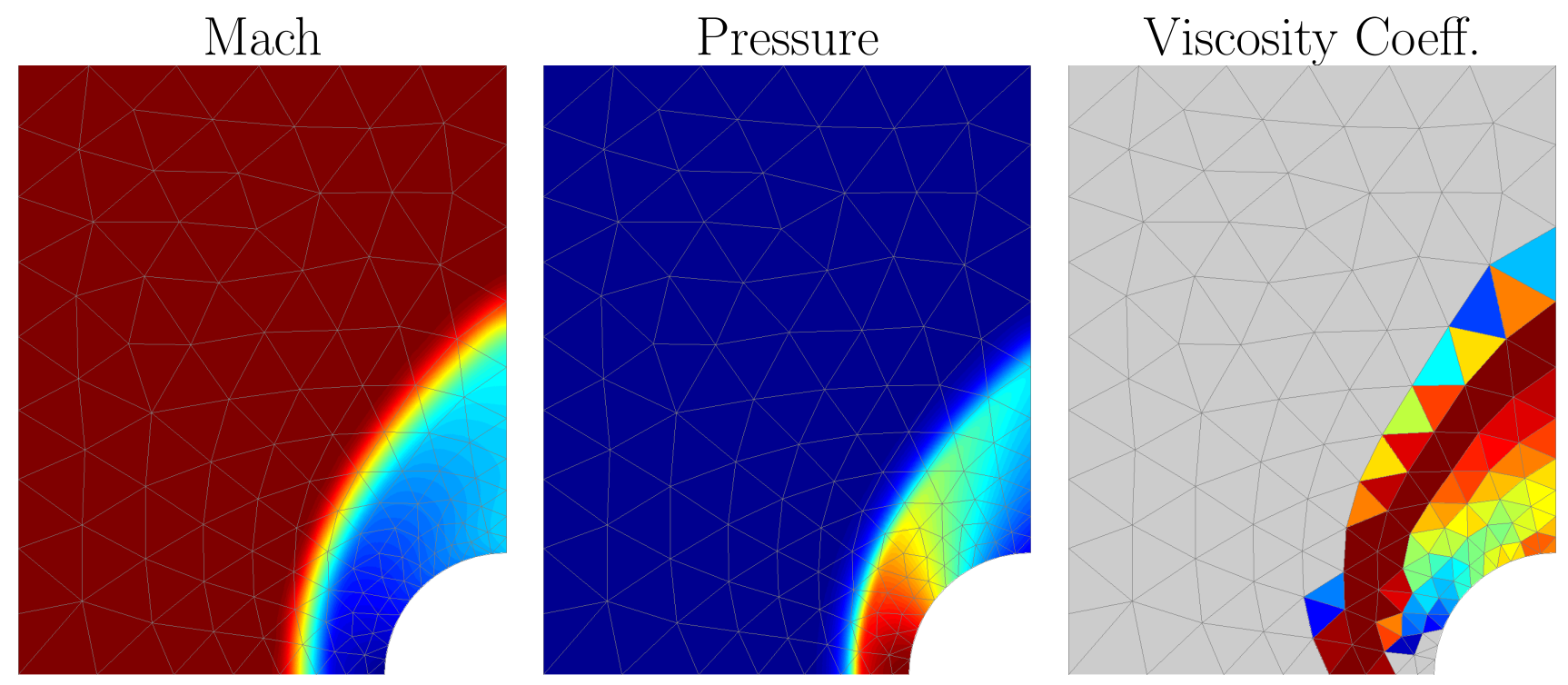

Figure 6. Mach 5 flow around a cylinder, with interpolation of degree $p=5$. Again, we observe that the shocks are resolved within the elements. 